\title{
Star Formation and Threshold in Nearby Galaxies Observed with GALEX
}

\author{
Samuel Boissier and the GALEX team
}

Laboratoire d'Astrophysique de Marseille

\begin{abstract}
Star formation on galactic scales is the process driving the evolution of galaxies. It is important to understand its various aspects in the nearby universe to properly interpret high redshift observations, and to construct correct models of the evolution of galaxies. Combining UV (GALEX) and infrared (IRAS) data for 43 nearby spatially resolved galaxies with corollary data (HI,CO), we study the star formation law ("Schmidt" law). The absence of a "threshold radius" in the UV supports the idea that star formation proceed at low gas densities and that the usual threshold observed in $\mathrm{H}$-alpha profiles is a small-number statistic effect.
\end{abstract}

In 43 nearby galaxies resolved by IRAS and observed with GALEX, we found that the star formation rates derived from the UV follows the Kennicutt 1998 "Schmidt" law, but extend to much lower surface densities (see lef Fig., where the gray area is the usual gas density threshold and our results are given with or without applying an extinction $\mathrm{A}(\mathrm{FUV})$ correction). We do not find a threshold radius in the UV, what suggests that the $\mathrm{H} \alpha$ threshold of Martin and Kennicutt (2001) is the radius where the number of ionising stars within an annular ellipse at any given time is so low that chances to measure a profile become vanishingly small (see right Fig., left axis: star formation rate within an annular ellipse, right axis: number of ionising stars within the same area). These results will be presented extensively in Boissier et al. (2006).
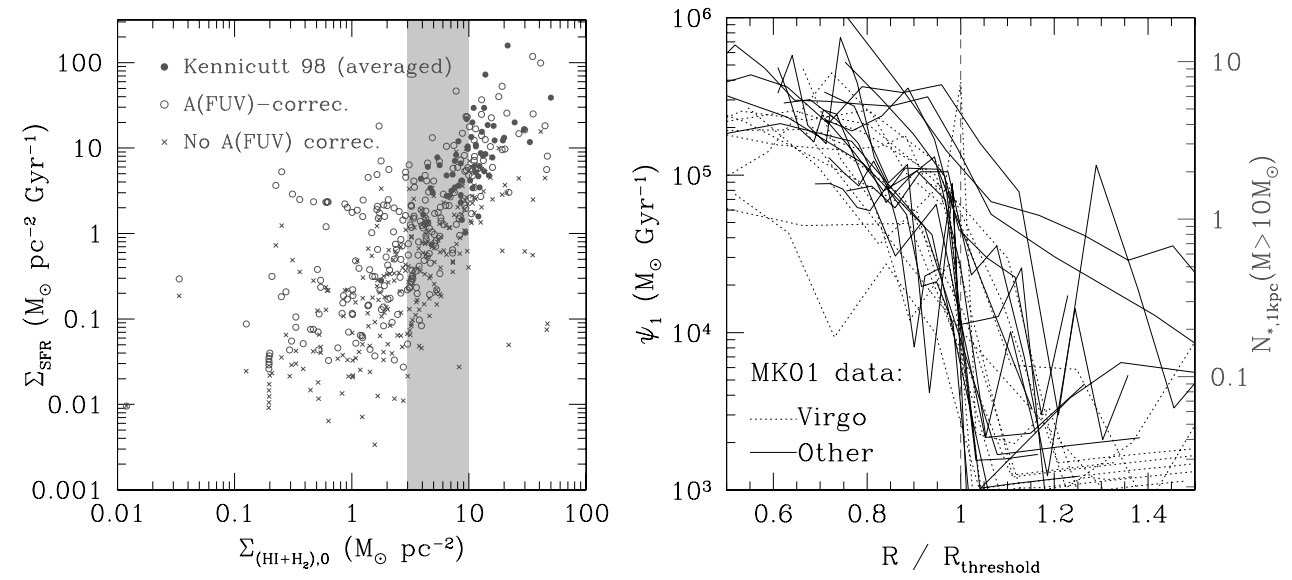

Acknowledgements: this work was done within the GALEX science team, with special input from A. Gil de Paz, A. Boselli, B. Madore, V. Buat, L. Cortese, D. Burgarella, J.-C. and Muñoz Mateos. We thank C. Quentin for her help in preparing and printing the poster.

\section{References}

Boissier et al. 2006, ApJS GALEX issue.

Kennicutt, R. C. 1989, ApJ 344, 685.

Kennicutt, R. C. 1998, ApJ 498, 541.

Martin, C. L., \& Kennicutt, R. C. 2001, ApJ 555, 301. 